\title{
A case of undetected congenital diaphragmatic hernia in an adult
}

\author{
S.J Jayasinghe, MBBS, MS (Colombo), MRCS (England) ${ }^{1}$, D.S. Liyanarachchi, \\ MBBS, FRCS ${ }^{2}$, \\ 1 Senior Registrar, National Hospital of Sri Lanka. \\ 2 Consultant Surgeon, National Hospital of Sri Lanka.
}

Key words: Congenital Diaphragmatic Hernia;

Haemopneumothorax; IC Tube Insertion.

\section{Introduction}

A27 year old female in septic shock was referred to the emergency general surgical team at the National Hospital of Sri Lanka (NHSL) from the Cardiothoracic Intensive Care Unit (ICU). Previously she had been treated at a general hospital for intrauterine foetal death and an intercostal (IC) tube has been inserted to the left chest on the grounds of an air-fluid level in the chest Xray. She had been transferred to the cardiothoracic ICU, NHSL 3 days later due to general deterioration with ongoing sepsis.

A CT scan was performed on clinical suspicion of the IC tube being inserted to the stomach in a patient with possible congenital diaphragmatic hernia. Oral CT contrast was leaking through the IC tube, and the CT scan confirmed the IC tube was in the stomach.

Emergency laparotomy revealed a $10 \times 10 \mathrm{~cm}$ sized diaphragmatic hernia with stomach, small bowel and spleen in the left thoracic cavity. Thoracic and peritoneal cavities were contaminated with gastric contents. The anterior wall of the stomach was found to be totally damaged by the IC tube.

The small bowel was brought to the peritoneal cavity after lengthy adhesiolysis and splenectomy was performed since it was unsalvageable. The anterior wall of the stomach was repaired. The diaphragmatic hernia was repaired with non absorbable suture material after extensive thoracic and peritoneal lavage, and two IC tubes were inserted. The abdomen was closed after inserting a drain.

Correspondence: S.J. Jayasinghe, Department of General Surgery,

Singleton Hospital, Sketty Lane, Swansea, UK, SA28QA

Email: vihangacj@yahoo.com

The Sri Lanka Journal of Surgery 2012; 30(1):39-40.
The patient was managed in the surgical ICU during the next 2 weeks. Limited re-laparotomy was performed for a leak from gastric repair and a gastrostomy tube was inserted.

She was treated with parenteral nutrition for 2 weeks and was discharged from hospital after a further 3 weeks.

\section{Discussion}

The improper fusion of the postero-lateral foramen of the diaphragm was first described by Bochdalek in 1848. The incidence of congenital diaphragmatic hernia varies from 1:2000 to 1:5000 live births and Bochdalek hernias account for 75 to $85 \%$ of these. Although it is a well-known entity in neonates, it is occasionally discovered incidentally in adult patients. [1]

The case of a 29-month-old child with a Bochdalek hernia presenting as a acute respiratory failure with chest radiography showing a tension gastrothorax that was misread as a tension pneumothorax has been reported. [2]

Our patient probably had an asymptomatic Bochdalek hernia, which lead to a diagnostic dilemma resulting in insertion of an intercostal tube into the stomach which was misidentified as a haemopneumothorax in the preoperative $\mathrm{X}$-ray. This had lead to septic shock, and the emergency laparotomy and repair was life saving. A detailed literature survey did not reveal any previous case reports similar to this in an adult. We are reporting this particular case in order to highlight the importance of considering all possibilities before making a 


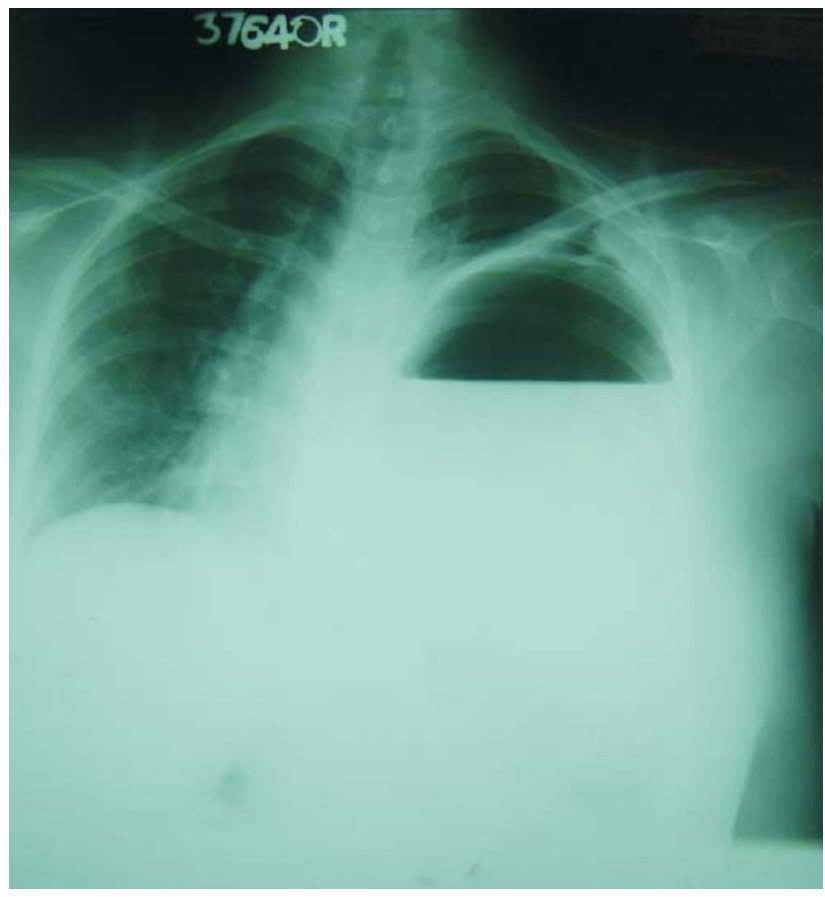

Figure 1. Chest X-Ray before laparotomy

decision to perform a basic surgical procedure like chest tube insertion.

\section{References}

1. Kumar A, Maheshwari V, Ramakrishnan TS, Sahu S. Caecal perforation with faecal peritonitis unusual presentation of Bochdalek hernia in

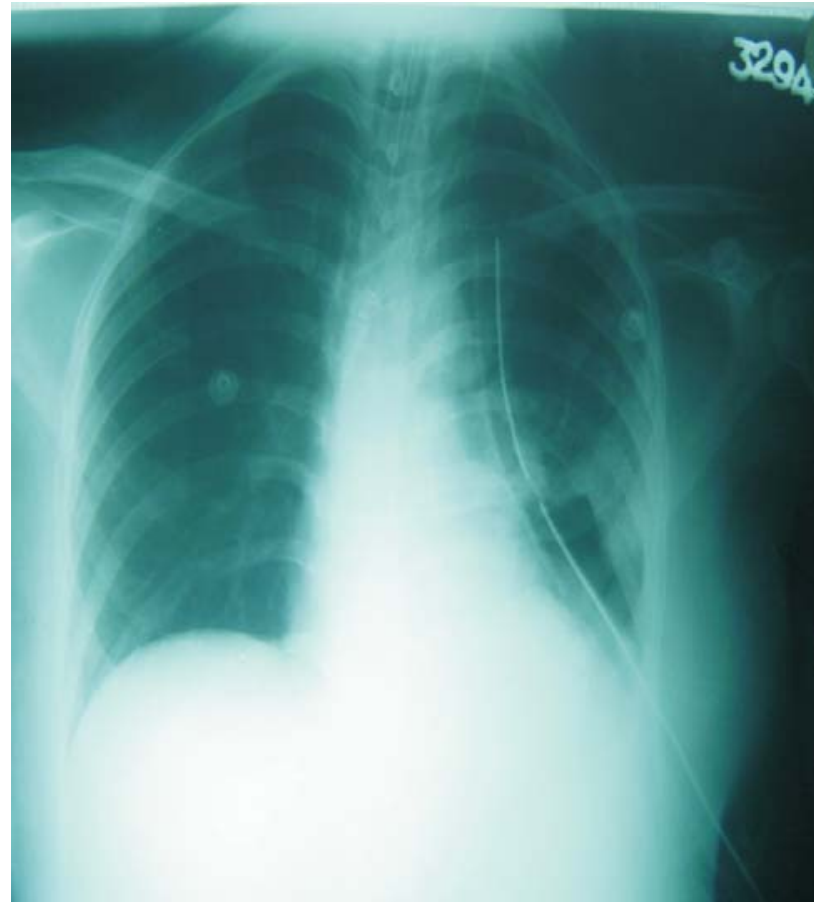

Figure 2. Chest X-Ray after laparotomy

an adult: a case report and review of literature. World J Emerg Surg. 2009; 4: 16 Published online 2009May 6. doi: 10.1186/1749-7922-4-16

2. Snyder HS, Salo DF, Kelly, PH. Congenital diaphragmatic hernia presenting as massive gastrothorax. Annals of Emergency Medicine 1990; 19(5); 562- 564.

\section{Key Learning Points}

- A congenital diaphragmatic hernia may be misidentified as a haemopneumothorax in an X-ray.

- IC tube insertion in such a patient can lead to catastrophic consequences. 